\title{
Design of a Second Generation Structural Column Implementing the Geometry of an Egg
}

\author{
Nischal Khawas ${ }^{1}$, Soumyadeep Sarkar ${ }^{2}$ \\ ${ }^{1}$ Research Scholar, Civil Engineering, Kurukshetra University, Kurukshetra, Haryana, India \\ ${ }^{2}$ Research Scholar, Electrical and Electronics Engineering, Kurukshetra University, Kurukshetra, Haryana, India
}

\begin{abstract}
A column or pillar in structural engineering is a structural element that transmits, through compression, the weight of one structure above other structure below. A stanchion that carries the load downwards to a foundation must possess the way to transfer the load without overstressing the foundation material. This paper presents a model for an egg shaped concrete confined by elliptical steel frames with binding bars. The benefits of hollow structural sections from a design and structural point of view have long been investigated by architects and engineers worldwide. This paper documents the design, operation and construction which are used to illustrate prospective applications, heuristic and empirical suggestions, technically made regarding construction of such a technique.
\end{abstract}

Keywords: Column; Egg shaped geometry; Golden Ratio; Granville’s Egg; Ellipse; bearing pads

\section{Introduction}

Columns are basically structural supports which are mainly used to sustain beams and arches where the upper parts of the wall or ceiling rest accordingly. In the early age pilasters and buttresses are usually constructed out of a stone or a monolith. They are placed, created, engineered and designed using multiple sections of stone, mortared or dry-fit together against each other. The design of the most ancient pillars assimilates the idea of entasis as enlightened by the roman historian, architect and engineer, Marcus Vitruvius Pollio. Most columns emerged from a base that rests on the top step of the crepidoma, except for the ancient Greek Doric column which commonly lies on the stylobate itself. Modern column maybe crafted out of steel or pre-casted concrete or left unembellished in a architectural housing.

Furthermore, all the structural components that exist, embrace the structure has different functions during their life time. The technique of design and construction is quite different for different structural elements. Not only the columns, fundamentally, all the structural elements are engineered and crafted for stress, flexure, axial forces such as tension compression, shear, torsion etc.

The propensity of a bridge to function depends directly on the persisting capacity of the bridge column to carry load. As far as the load carrying capacity of a column is concerned, structural columns can fail, or collapse, in either by crushing or by buckling. Crushing supervenes when the weight of any distributed load simply exceeds the compressive strength of the material from which the column is devised. As columns are customarily fabricated from dense materials such as stone, concrete, or steel, this type of failure is relatively deviant. Also structural columns are relatively studied and researched by civil engineers worldwide to make it as efficient as possible to prevent any jeopardy. They are also organized and systematized on the basis of its parameters which are likely applicable and definable.

Therefore, the basic convention which defines the structure either must be able to suitably fulfill its justification id est the serviceability and prerequisite ability to safely support and resist its design loads.

The ultimate goal of this research is to capture the degradation of strength, increase efficiency in analytical models that can be applied to more complex bridge and establishment of structures.

\section{A. Classifications of Structural Columns with its Respective Parameters:}

Structural columns can be efficiently systematized on the basis of its parameters viz. slenderness ratio, shape, type of loading and motif of its lateral reinforcement.

- Classification based on Ratio of Slenderness:

The column can be classified in terms of Short Columns and Long or Slender Columns. The ratio of the effectual operative length of a column to the least radius of gyration of its cross section is called the slenderness ratio. It's denoted by $\lambda$ (Lambda). It means that if the length is greater than the critical buckling length and it will fail due to buckling of the column.

- Classification based on shape:

The column can also be classified in terms of its shape such as Rectangle, Square, Circular, Polygon and T, L, or + shapes Columns. To add, if the length of the column is less than the critical buckling length, it will result in jeopardy due to shearing.

- Classification based on types of loading:

The columns can be further classified in terms of loading which manifests, axially loaded column, axial load and un-axial bending column, axial load and biaxial bending column

- Based on pattern of lateral reinforcement:

In terms of Spiral Columns and Tied Columns, it can be classified based on the pattern of lateral reinforcement. Spiral columns are basically cylindrical columns with a continuous helical crosspiece wrapping around the column and Tied Columns are closed lateral ties spaced about uniformly across the column.

Additionally, the increased stresses due to the combined

\section{Volume 5 Issue 11, November 2016}




\section{International Journal of Science and Research (IJSR) \\ ISSN (Online): 2319-7064}

Index Copernicus Value (2015): 78.96 | Impact Factor (2015): 6.391

axial-plus-flexural stresses result in a reduced load-carrying ability. And as far as this research is concerned, the article implements the idea of coupling of golden ratio of an egg with bridge columns. Designing the structure of the column in such a way that it requires fewer amounts of material which is equally high load bearing capacity and cost effective in nature. The structure of an egg is designed and fabricated by the nature in such a way that when vertical force is applied at its ends, the egg sustains the pressure and prevents itself from breaking. The reason why it doesn't break, although the egg shell is such a brittle material, redirects to the mathematical explanation and construction of the structure. Briefly, it can be presented and described by the idea of equal distribution of pressure throughout its structure creating maximum number of load bearing points on its body which absorbs such a high amount of pressure making it indestructible at certain amounts of pressure.

\section{What is Golden Ratio?}

Golden Ratio is an irrational number and its value is

1.6180339. It is also called the Golden Mean, Golden section, Divine Proportion, etc. which is represented by a Greek letter phi $(\varnothing)$.

$$
\frac{a+b}{a}=\frac{a}{b} \stackrel{\text { def }}{=} \varphi, \quad \underbrace{a}_{\begin{array}{c}
a+b \\
a+b \text { is to } a \text { as } a \text { is to } b
\end{array}}
$$

This Golden number 1.6180339 is found by dividing the line in such a way that the ratio of the whole part to the longest part is same as dividing the longest part to the smallest part and this irrational number goes up to infinity, as with pi $(\pi)$ also an irrational number which is 3.147; which is the ratio of the circumference of the circle to its diameter. This fascinated number has been used since the ancient times. A Greek sculptor and mathematician, Phidias, has applied this number phi $(\varnothing)$ to the design of Parthenon. The construction of a pentagram also relates to the golden ratio constructed by Euclid. Later, Plato also considered this ratio as the universal binding of mathematical relationships. Fibonacci sequence discovered by mathematician, Leonardo Fibonacci, relates to the golden ratio. This sequence of series $0,1,1,2,3,5,8,13$, $21,34,55,89,144,233$, and so on is closely related to the golden ratio when the preceding number is divided by the succeeded number. Further the series goes higher and higher, the closer is the value nearer to the golden ratio. This Fibonacci series is obtained by adding the first number to the second number and goes on and on.

\section{- General Quadratic Mathematical Equations of Phi}

$(\varnothing)$ :

$$
\emptyset^{2}-\emptyset-1=0
$$

\section{- Uniqueness of the value Phi $(\varnothing)$ :}

When this number is squared, the resultant value will be greater than itself by 1 i.e. 2.618 and its reciprocal value will be less than the number by 1 , which is 0.618 .

\section{- Geometry of the Golden Ratio:}

The Golden ratio appears in the construction of geometry and it is visible in Equilateral triangle, square and pentagon when put inside a circle. Other Golden geometrical shapes include Golden triangle, rhombus, rectangle, pentagon and pentagram. Three-dimensional solids include dodecahedrons, icosahedrons, triacontahedron whose faces are in golden ratio.

\section{Relationship of the Golden Ratio with the Structure of An Egg}

The structure of an egg is enormously related to the golden ratio and many architects have tried to derive and construct the hidden secrets of the structure of an egg which is in relation to the golden ratio. Many geometrical construction methods are possible for the design of an egg. Using simple circular arcs, one can construct the planar structure of an egg exactly without the use of Mathematical formula. Some of the methods used in the construction of the golden egg are:

1) The Construction by the Method of $45^{\circ}$ Angle.

2) The Construction Using the Regular Pentagon

3) The Construction Using the Penrose Tiling Triangle

The basic and simple construction of the egg structure is done by the method of 45-degree angle and this construction method can be used for the practical design of an egg shaped column.

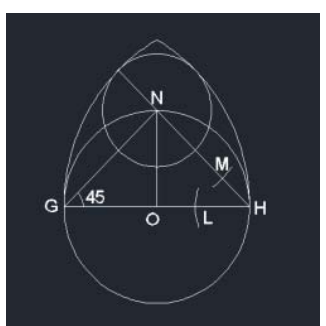

Figure 1: Construction of golden egg by 45 angle method

Consider any value of radius ' $r$ ' to be the semi-circle of radius' $r$ ' with center ' $O$ ' as shown above in fig (1). From the end point ' $\mathrm{H}$ ' and ' $\mathrm{G}$ ' of the semi-circle, an arc having radius $2 \mathrm{R}$ is drawn opposite to each point and then a 45-degree angle is drawn, from the points $\mathrm{H}$ and $\mathrm{G}$. The 45-degree angle line intersects at the point $\mathrm{N}$. The length of 'NO' must be measured to bisect the 45-degree line at point ' $M$ ' and from the point ' $M$ ', line ' $O H$ ' must be bisected at point ' $L$ ' taking the length 'MH'. Then the length of 'LH' must be measured, which in turn will be the radius of the circle centered at point $\mathrm{N}$, which further touches the arc on both the sides produced from point ' $\mathrm{G}$ ' and ' $\mathrm{H}$ '.

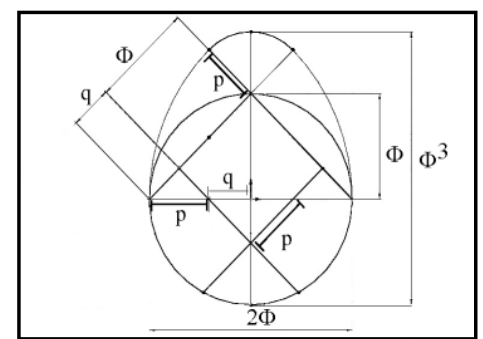

Figure 2: Properties of the golden egg

The planar geometry of the egg shape showing unique properties in terms of golden ratio in the above figure.

$$
P=\frac{2 \emptyset}{2+\sqrt{2}}
$$




$$
q=\frac{\emptyset}{1+\sqrt{2}}
$$

$$
p+q=\emptyset
$$

\section{Granville's Egg Curve}

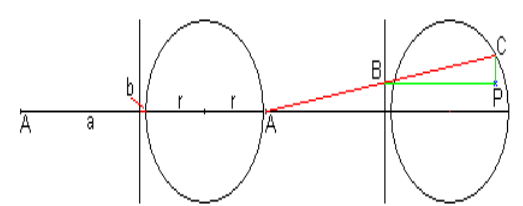

Figure 3 (a): Granville's Egg

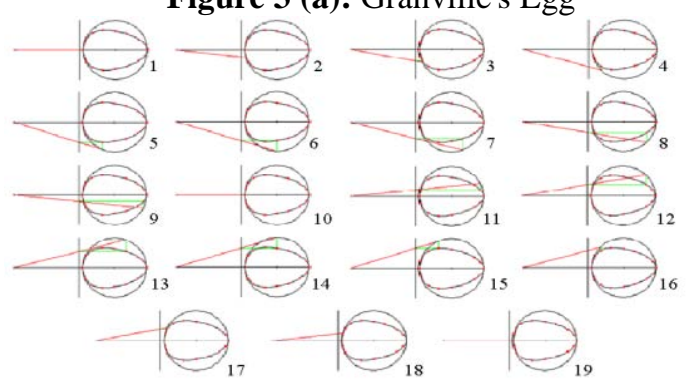

Figure 3 (b): Phases of the Granville's egg curve

Granville's egg curve has shown that the egg shape curve can be drawn using a circle and two lines perpendicular to each other. Considering a horizontal line bearing one end ' $\mathrm{A}$ ' and a vertical line which is perpendicular to the horizontal line at a distance ' $a$ '. A circle is drawn at a distance of $(a+b)$ as shown in fig. (3a), having radius ' $r$ ', whose diameter lies on the horizontal line i.e., symmetric about the horizontal line. Any line projecting from the point ' $\mathrm{A}$ ' towards the circle cuts the circle at point ' $\mathrm{C}$ ' and the vertical line at point ' $\mathrm{B}$ '. If a horizontal line is projected from point ' $\mathrm{B}$ ' and vertical line from point ' $\mathrm{C}$ ', the projected lines meet at $\mathrm{P}$. When the point ' $\mathrm{C}$ ' moves on the circle, the point ' $\mathrm{P}$ ' describes an egg shaped curve. Figure ( $3 \mathrm{~b}$ ) given above presents multiple phases of the completion of an egg shape curve using Granville's egg construction method.

\section{Mechanical Construction of the Curve of An Egg}

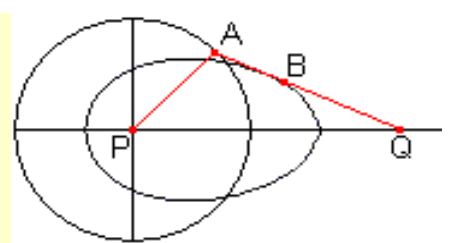

Figure 4(a): Mechanical construction of an egg.

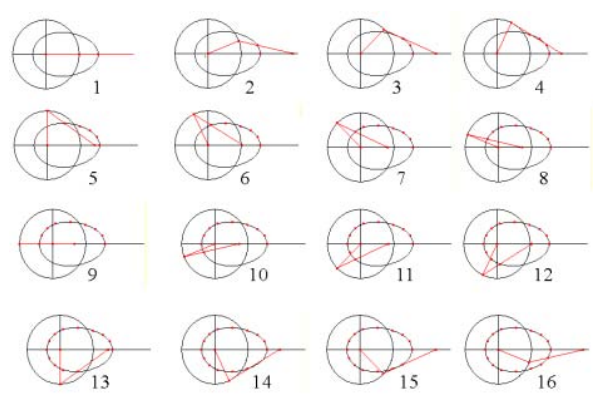

Figure 4(b): Phases of the Mechanical Construction of the egg curve

Consider a fixed point ' $\mathrm{P}$ ', which is the center of a circle as shown in fig. (4a)

The point ' $\mathrm{A}$ ' moves around the circle having radius $\mathrm{R}=\mathrm{PA}$. And he point ' $Q$ ', which lies outside the circle, moves forth and back horizontally. The line ' $\mathrm{QA}$ ' is joined and point ' $\mathrm{B}$ ', which lies on the line ' $\mathrm{QA}$ ', traces the shape of an egg shaped curve, when the point ' $A$ ' moves on the circumference of the circle.

Figure (4b) shows the phases of an egg curve formed. Initially, it is started when the point ' $\mathrm{A}$ ' lies horizontal to ' $\mathrm{P}$ ' and completes half part of the curve, when the point ' $A$ ' completes 180-degree and finally full curve is traced when the point ' $\mathrm{A}$ ' traverses 360-degree.

Applying Mathematical equations, the curve of the egg shape can be obtained. Many mathematical equations have been derived. Modifying the equation of an ellipse, the Egg Shape can be drawn. The ellipse is symmetrical about both the axes as it can be seen from the basic formula of an ellipse (x2/a2 + y2/b2 = 1). An ellipse is commonly used in Engineering, Physics and Astronomy. It is derived from the cross -section of a conic section unless the section is parallel to the axis of the cylinder. It has two focal points F1 and F2. Major and minor axes are represented by 'a' and 'b' as shown in fig (6). At any point, the sum of the distances from the two focal points is equal to twice the major axis ( $\mathrm{PF} 1+\mathrm{PF} 2=2 \mathrm{a})$. Ellipse has an eccentricity ranging from 0 to 1 . The eccentricity, generally denoted by 'e', is a constant value, which associates the conic section and determines how much the conic section is deviated with respect to the circular curve.

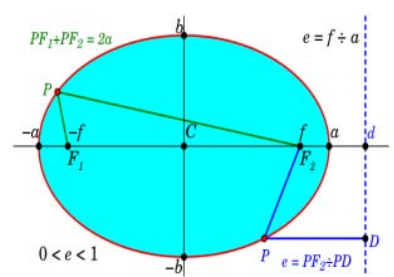

Figure 5: Diagram of an ellipse

When the basic equation of the ellipse as given above is modified, the oval or the elliptical curve changes to egg shape curve. The elliptical equation is modified by multiplying $\mathrm{y}^{2}$ by a suitable term $\mathrm{n}(\mathrm{x})$. This $\mathrm{n}(\mathrm{x})$ term makes the value of $(\mathrm{y})$ larger on the right side of the $y$-axis and smaller on the left side. So, the curve of the ellipse changes to egg shape. 


\section{International Journal of Science and Research (IJSR) \\ ISSN (Online): 2319-7064}

Index Copernicus Value (2015): 78.96 | Impact Factor (2015): 6.391

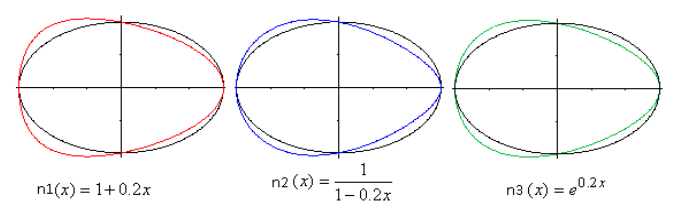

Figure 6: Equation of the curve.

Addressing to the red colored egg shape from the figure (6), the $\mathrm{n} 1(\mathrm{x})$ term is equal to $1+0.2 \mathrm{x}$. As seen from the equation above, when this term is multiplied, the minor axis of the ellipse changes and the curve on the right side lies inside the ellipse while the left side of the curve lies outside the ellipse.

Now, comparing the blue and green egg shaped curve, the multiplying terms $\mathrm{n} 2(\mathrm{x})=1 /(1-0.2 \mathrm{x})$, follows a geometric series which is given by $1 /(1-0.2 x)=1+0.2 x+0.04 x^{2}+\ldots$, and $\mathrm{n} 3(\mathrm{x})=\mathrm{e}(0.2 \mathrm{x})$ follows a Taylor's series which is given by $\exp (0.2 \mathrm{x})=1+0.2 \mathrm{x}+0.02 \mathrm{x}^{2}+\ldots$

Now, Taylor series, in which we also defined the above equation is nothing but an infinite number of sums providing the value of a function in the neighborhood of a point ' ' in terms of the derivatives of the function evaluated at . Geometric series as we described above is an infinite series in mathematics in which the ratio of each two consecutive terms is a constant function of the summation index. The general equation of the geometric series is written as $a+a r+a r 2+a r 3+\ldots$, where ' $a$ ' is the first term of the series and ' $r$ ' is the constant ratio.

\section{Bearing Pads}

Bridge decks without the support of bearing pads are liable to damage due to vehicular movement and heat expansion of the decks. Bearing Pads provide a connection between super-structure and sub-structure and controls the interconnection of loading and movements between parts of a structure. Bearing pads also provides a uniform transfer of load from beam (bridge decks) to sub-structure (bridge column/ pier). Bearing pads are used widely in bridges and buildings. These are manufactured from quality natural rubber and neoprene.

\section{Functions of Bearing Pads:}

1) It supports vertical loads.

2) Transmitting load to the sub-structure foundations.

3) It mainly allows bridge deck rotation.

4) Allows lateral and rotational movements due to dynamic forces like Seismic and Wind. Shrinkage and creep due to temperature changes and even vibrations due to vehicular movements.

\section{Types of Bearing Pads:}

\section{A. Laminated Elastomeric Bearing Pads (Steel Reinforced)}

Laminated elastomeric bearing pad allows the movement of the bridge deck horizontally and accommodates even the rotational movement about any axis by elastic deformation. They are commonly used in modern bridges where the bridges are susceptible to high loading, excessive translation and rotations. Elastomers, as used in a bearing pad, is a compound manufactured from a high quality natural rubber and to attain the desired load bearing capacity, steel and rubber are alternatively processed together. Laminated bearings can be rectangular or circular as per the requirement of the bridge type. It is ideal and economical for the buildings and large span bridges.

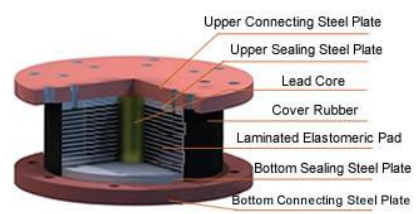

Figure 7: Laminated Elastomeric Bearing Pad.

\section{B. Sliding Bearing Pads (Frictionless Bearing Pads)}

Sliding Bearing Pads are used where the relative translation is high with respect to the area in the abutment. This type of bearing pad is commonly used in airport and malls, where the relative translation is very high. PTFE (Polytetrafluoroethylene) sheets with stainless steel plates are used in this bearing pad to obtain the desired result for high translation. At the top portion of the bearing pad PTFE is chemically bonded or fixed using adhesives.

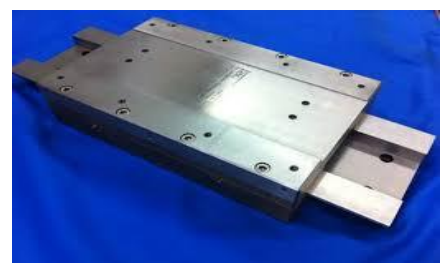

Figure 8: Sliding Bearing Pad

\section{Pot Bearing Pads}

Pot Bearing Pads are designed to bear heavy loads and allow rotation and translation within the enclosed area of the bearing pad. This Pot bearing pad is used extensively in the bridges where the rotation is very high in the bearing pad. Under loading, the bearing pad can be deformed but it is incompressible and has a capacity to withstand heavy vertical loads. It is mainly three types.

a) Fixed Pot Bearing: It constraints longitudinal and transversal movements.

b) Free Sliding Pot Bearing: It allows longitudinal as well as transversal movements.

c) Guided Pot Bearings: It allows longitudinal movement but constraints transversal movements.

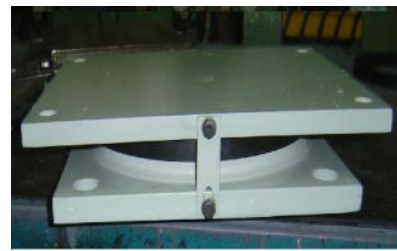

Figure 9: Pot Bearing Pad

\section{Disc Bearings}

Disc Bearings are used mainly in the railroad and highway bridges. This type of bearings is used where the effect of live loads is very high. The Polyurethane Disc element present in the bearing provides cushioning effect and durability, thus bearing excessive live loads. It is commonly present in the rail road and has advantages over Pot Bearings when it is subjected to excessive cyclic loading, as it possesses high fatigue resistance properties.

\section{Volume 5 Issue 11, November 2016




\section{International Journal of Science and Research (IJSR) \\ ISSN (Online): 2319-7064}

Index Copernicus Value (2015): 78.96 | Impact Factor (2015): 6.391

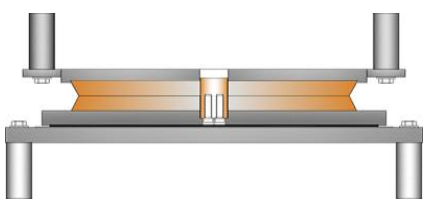

Figure 10: Pot Bearing Pad.

\section{Working and its Implementation}

The Egg Shape which is in accordance to the Golden Ratio can be applied to the column. The design of this Egg Shaped Column can be useful especially in the Rail Road Bridges and Highway Bridges. Bridge Column is also termed as a Pier.

Piers are generally a vertical column raised above a water body, supported by Pile Foundation. Bridges and Buildings are commonly supported by Piers. The use of pier (bridge column) makes the structure open above the water body level and allows the tides and hurricanes to pass through it and ships to navigate, without obstruction and above the ground level of the Highway Bridges, it allows for the passage of vehicles, humans, etc. in urban areas and wild animals to pass safely through it in the forests. A pier ranges in size from a simple wooden structure to complex major structures. Piers are built for different purposes and they differ in the size and design.

Despite the fact, that egg shell being so brittle in nature, can bear high compressive force, if applied on the top and bottom of the egg, vertically. When the compressive force is applied on the top of the shell, the curve of an egg being smooth and edge- free, the force gets evenly distributed throughout the whole surface of the shell making it almost indestructible.

The followed principle is particularly same, when applied to the structural element, column, for any structure. Highway Bridge and Rail Road Bridge Columns are among the columns which are mostly drafted out of concrete. Not just simple ordinary concrete, but reinforced concrete.

To briefly describe, what is concrete, it is nothing but a combination made of cement, sand, aggregates and water which are widely used to build any structure. The property of concrete is such that, it can bear high compressive loads or forces. Depending upon the grades of concrete, the stress (compressive load) taken by the concrete block varies. As per the Indian Standard specification ( of concrete are given below in Table 1 :

Table 1: Grades of Concrete

\begin{tabular}{|r|l|l|l|l|l|l|l|l|l|}
\hline \multicolumn{10}{|c|}{ Grades of Concrete } \\
\hline Grade Designations. & M15 & M20 & M25 & M30 & M35 & M40 & M45 & M50 & M55 \\
\hline $\begin{array}{c}\text { Characteristic strength } \\
\text { at 28 days in MPa. }\end{array}$ & 15 & 20 & 25 & 30 & 35 & 40 & 45 & 50 & 55 \\
\hline
\end{tabular}

The letter ' $M$ ' refers to the mix and the number specifies the characteristic strength of $150 \mathrm{~mm}$ cube after 28 days and it is expressed in $\mathrm{MPa}(\mathrm{N} / \mathrm{mm} 2)$.

Now, ricocheting back to the topic, as it would mention, in spite of the concrete being hard and can take high compressive loads, steel rods have to reinforced to make the structure more stable. If we examine a beam, casted out of only concrete, it cannot resist heavy loads under flexure. When the beam is loaded, and load is increased, it deforms and attains the shape of a circular curve.

During the process of deformation, the upper part above the neutral axis of the beam will be in compression and the lower part below the neutral axis will be in tension. As the concrete can withstand only compressive loads, it cannot resist the tension in the lower part of the beam and fails by cracking. So, the steel rods need to be reinforced as it can withstand high tensile force. The lower part i.e., below the neutral axis of the beam, must be reinforced with steel rods so that the beam can resist heavy loads.

Therefore, in the design of an egg shaped column same principle is drawn from the bending of a beam. If we keep on increasing the axial load on an egg shaped column, vertically i.e. on the top portion, the outer part of the column will be in tension and the inner part will be in compression. This brings to the conclusion, that the outer part must be reinforced with steel bars so that it can resist heavy loads and thus preventing it from cracking or complete failing. Stirrups are provided for the shear stress and binding it in the form of Spiral shape will make the column more stable and economical design, when the egg column is casted hollow. If computed and analyzed using software, the material used i.e. concrete and the steel, in the egg shape column would be significantly less than the material required in the ordinary design of columns. Even the lower grade of concrete and providing less number of steel rods to be reinforced, the egg shape column can bear high load and further reduces the dead load imposed on the footing and foundation, thereby saving the cost of the foundations.

\section{Conclusion}

Designers have been designing the columns in different shapes as per the design load and for the aesthetics. Columns are being improved in the design, to enhance performance and making it cost effective. Hollow columns were designed later, which proved to be economical than the solid columns, though the cost of extra form work may differ from the material savings.

The egg shaped column, where the golden ratio resides well, can be best suited for short and intermediate height columns. This egg curve shape can be easily obtained and even with high accuracy by using a computer. The program must be coded in such a way that, by just inputting the value of the semicircular radius, in the case of the 45-degree construction method, it automatically generates a curve of the egg shell. Other egg shape curves can also be obtained easily with the help of computer.

As it is said that the egg shaped column will require fewer materials as compared to the ordinary columns, prima facie, these will have a major impact on its efficiency and making it less expensive to build. So, basically the convention which defines the entire structure either must be able to suitably fulfill its premise, which is the serviceability and prerequisite ability to safely support and resist its loads placed above the structure.

\section{Volume 5 Issue 11, November 2016}




\section{International Journal of Science and Research (IJSR) \\ ISSN (Online): 2319-7064 \\ Index Copernicus Value (2015): 78.96 | Impact Factor (2015): 6.391}

Fiber Reinforced Polymer (FRP) composites, can also be used in the construction of an egg shape column. Glass Fiber Reinforced Polymer (GFRP) and Carbon Reinforced Polymer (CFRP) can be used as a reinforcing material. This makes the column light weight, equivalent strength, low maintenance and durable in extreme environmental conditions. It can also be pre-casted as per the requirement. By the method of Pre-tensioning, during the process of pre-cast, the strength increases in comparison to the ordinary reinforced egg shape column.

Therefore to conclude this article, this research may prove valuable details regarding designing of piers and increase efficiency in analytical models that can be applied to more complex bridge and establishment of structures.

\section{Future Work}

The detailed research and mathematical verification of this research will be minutely scrutinized and will be followed subsequently.

\section{Acknowledgement}

I would like to thanks my parents for sharing their pearls of wisdom and supporting this research and I would also like to give sincere gratitude especially to Mr. Soumyadeep Sarkar for his guidance and support to pursue this work.

\section{References}

[1]

available

www.researchgate.net/publication/273139544

[5] , available at www.wikipedia.com

[6] , available at www.wikipedia.com

[7]

[8]
, available at www.bridge.orf.ae , available at www.mi.sanu.ac.rs available at www.goldennumber.net , available at www.wikipedia.com

\section{Author Profile}

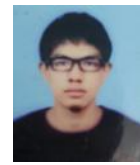

Nischal Khawas is currently pursuing B. Tech in Civil Engineering from Kurukshetra University, Kurukshetra. He is a research scholar and also a member of American Society of Civil Engineers (ASCE). His interested areas of research are Structural Designs, Structural Analysis, Geotechnics, Fluid Mechanics and Transportation Engineering.

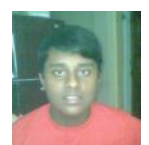

Soumyadeep Sarkar is currently pursuing B. Tech in Electrical and Electronics Engineering from Kurukshetra University, Kurukshetra. He is a research scholar and also an eminent member of The Institute of Electrical and Electronics Engineers (IEEE). His interested areas of research are Electrical Machines and Drives, flexible AC transmission systems, High Voltage AC and Power Electronics. His other research interests include Quantum Physics, Biomedical Science, Structural Engineering, Classical Mechanics, Astrophysics and Biological Psychiatry. 